\title{
Lymphangiogenic factors, mechanisms, and applications
}

\author{
Wei Zheng, Aleksanteri Aspelund, and Kari Alitalo
}

Wihuri Research Institute and Translational Cancer Biology Program, University of Helsinki, Helsinki, Finland.

\begin{abstract}
Lymphangiogenesis, the growth of lymphatic vessels, is essential in embryonic development. In adults, it is involved in many pathological processes such as lymphedema, inflammatory diseases, and tumor metastasis. Advances during the past decade have dramatically increased the knowledge of the mechanisms of lymphangiogenesis, including the roles of transcription factors, lymphangiogenic growth factors and their receptors, and intercellular and intracellular signaling cascades. Strategies based on these mechanisms are being tested in the treatment of various human diseases such as cancer, lymphedema, and tissue allograft rejection. This Review summarizes the recent progress on lymphangiogenic mechanisms and their applications in disease treatment.
\end{abstract}

\section{Introduction}

Lymphatic vessels form an elaborate vascular system throughout the skin and in most internal organs. They interdigitate with blood vessels and play important functions in interstitial fluid drainage, lipid absorption, and immune responses. It seems that most lymphatic vessels originate from veins during embryonic development and then undergo extensive expansion and remodeling to form a hierarchical, mature vessel network consisting of lymphatic capillaries, precollectors, and collecting vessels, which conduct lymph fluid through the LNs and back to the blood circulation (1). SOX18 and COUP-TFII transcription factors cooperate to activate PROX1, which marks cells within the embryonic veins that will differentiate to lymphatic endothelial cells (LECs) (2). Migration of $\mathrm{PROX}^{+}$cells away from the embryonic veins requires VEGFC (3). Subsequent lymphatico-venous separation is mediated by podoplanin, which triggers platelet aggregation to block the entry of blood into the emerging lymphatic vessels $(4,5)$. Lymphangiogenesis, or the growth of lymphatic vessels from preexisting vessels, is the major if not exclusive mode of lymphatic growth. Knowledge of the mechanisms regulating lymphangiogenesis has grown significantly since the discovery of lymphangiogenic growth factors and receptors over 15 years ago (6-8). This Review provides an overview of the growth factors and signaling mechanisms in lymphangiogenesis and of the recent advances in their application for therapeutic purposes in preclinical models and in the clinic.

\section{Lymphangiogenic pathways and mechanisms}

VEGFC/D and VEGFR3. Signaling via VEGFC/D and VEGFR3 is perhaps the most central pathway for lymphangiogenesis $(1,6)$. VEGFC is essential for the sprouting of ECs from embryonic veins that are committed to the lymphatic lineage. The first LECs in embryos sprout from the cardinal vein to form a lymphatic network during embryonic development $(3,9)$. A paracrine VEGFC gradient induces sprouting, and biallelic Vegfc deficiency is embryonic lethal (3). Vegf $c^{+/-}$mice are born alive but suffer from lymphatic deficiency and subsequent lymphedema (3). Similarly, inhibition of VEGFR3 signaling during the formation of lym-

Conflict of interest: Kari Alitalo is a scientific adviser for Laurantis Pharma and has a research grant from MedImmune Inc.

Citation for this article: J Clin Invest. 2014;124(3):878-887. doi:10.1172/JCI71603. phatic vessels induces lymphatic regression and lymphedema in mouse embryos and neonates (10). Interestingly, rare alternatively spliced transcripts encoding soluble forms of VEGFR2 (sVEGFR2) and VEGFR3 (sVEGFR3) may limit the local activity of VEGFR3 in some tissues, such as the cornea $(11,12)$.

A number of growth factors induce the dimerization and autophosphorylation of their tyrosine kinase receptors (13), which then serve as docking sites for downstream signaling molecules to coordinately regulate cellular responses (14). VEGFC-induced VEGFR3 activation leads to phosphorylation of the serine kinases AKT and ERK, which promotes LEC proliferation, migration, and survival (15). PI3K, the upstream activator of AKT, was found to interact directly with phosphorylated VEGFR3 and to mediate LEC tube formation and migration (16). Interestingly, while VEGFC induces strong ERK activation in both LECs and blood vascular ECs (BECs), it induces strong AKT activation only in LECs (17). Likewise, deletion of the PI3K catalytic subunit $P i k 3 c a$ or a regulatory subunit Pik3r1 in mice leads to defective lymphatic growth and maturation without a major blood vascular phenotype $(18,19)$. These findings suggest that AKT has a distinct function in LECs. Downstream signal transduction of VEGFR3 also involves the Rho GTPase Rac1 (20). Endotheliumspecific Rac1 deletion results in impaired blood-lymphatic vessel separation during embryonic development, and Rac1-silenced LECs fail to migrate toward VEGFC (20). The proteolytically processed form of VEGFC induces heterodimerization of VEGFR2 and VEGFR3 in LECs (21), but the signaling specificity of such heterodimers is incompletely understood.

Missense mutations in the tyrosine kinase domain of VEGFR3 are responsible for about $70 \%$ of cases of an autosomal dominant primary congenital lymphedema known as Milroy disease $(22,23)$. Additionally, a homozygous recessive mutation has been identified in a lymphedema patient $(24,25)$. The dominant mutants not only fail to respond to VEGFC stimulation, but also inhibit the activation of a cotransfected WT VEGFR3, whereas the recessive mutant showed reduced activation without the dominant negative activity $(22,24)$. A further screen of lymphedema patients has recently identified a frameshift Vegfc mutation that failed to induce lymphatic sprouting when overexpressed in zebrafish (26). Thus, evidence from mouse models and human patients indicates that the VEGFC/VEGFR3 signaling axis is the major driving force of lymphangiogenesis. 
VEGF and VEGFR2. VEGF/VEGFR2 signaling is less important than VEGFC/VEGFR3 signaling for lymphangiogenesis. Exogenous VEGF165 as well as VEGFE, a viral-derived VEGF that activates only VEGFR2, can induce lymphatic vessel enlargement but very little sprouting (27). In tumor xenograft models, VEGF overexpression can induce peritumoral lymphangiogenesis and $\operatorname{LN}$ metastasis $(28,29)$. However, secondary effects may be involved, as VEGF recruits VEGFC/D-producing macrophages to sites of its expression (30-32). Notably, VEGFE-activated VEGFR2 cannot rescue the lack of lymphatic vessels resulting from VEGFR3 blockade (27). It seems that VEGFA can restrict lymphangiogenesis indirectly via an endogenous trapping mechanism involving VEGFR2 upregulation in BECs with subsequent sequestration of VEGFC (33). On the other hand, VEGFCinduced angiogenesis is attenuated in the presence of abundant lymphatic vessels (34). These results suggest that the bioavailability of VEGFs also plays a role. Constitutive deletion of Vegf 2 from lymphatic vessels leads to hypoplastic but functional lymphatic vessels in embryos and adult mice (35). Thus, VEGF appears to be a weak lymphangiogenic factor.

A recent study has shed some light on the weak lymphangiogenic potential of VEGF. It was shown that lymphatic sprouting induced by VEGF is profoundly enhanced by concomitant inhibition of the Notch pathway, whereas the strong lymphatic sprouting induced by VEGFC was not increased by Notch inhibition (36). These data suggest that the Notch pathway restricts VEGF activity in LECs.

Collagen and calcium-binding EGF domain-containing protein 1. The secreted collagen and calcium-binding EGF domain-containing protein 1 (CCBE1) was recently identified as a highly conserved lymphangiogenic factor in zebrafish (37) and mice $(9,38)$. Similar to Vegfc-deleted embryos, embryos lacking Ccbe1 fail to form lymphatic vessels $(9,38)$. Furthermore, mutations in the CCBE1 gene have been identified as a cause of the Hennekam syndrome, a primary human lymphedema (39). CCBE1 alone induces little lymphangiogenesis but markedly enhances the lymphangiogenic activity of VEGFC (38). The mechanism of this molecular interaction is still unknown.

Semaphorins and neuropilins. Previously know for their importance in guiding axon migration in the CNS, semaphorins (SEMAs) and neuropilins (NRPs) have recently received much attention for their involvement in angiogenesis and lymphangiogenesis, as well as tumor growth $(40,41)$.

NRP2 is expressed in embryonic veins before lymphatic vessels are formed, and in lymphatic vessels at later stages (42, 43). Nrp2deficient mice have a grossly normal cardiovascular system, but the small lymphatic vessels and capillaries are absent or markedly reduced (44). VEGFC and VEGFD bind to NRP2 and induce its cointernalization with VEGFR3, although NRP2 complexes with VEGFR3 even in the absence of these ligands (45). An antibody that specifically blocks VEGFC binding, but not SEMA binding to NRP2 (46), inhibits VEGFC-induced VEGFR 3 activation and lymphatic sprouting in vitro and in neonatal mice $(46,47)$. These findings indicate that NRP2 is a VEGFC coreceptor that facilitates VEGFR3 signaling.

The NRP1 ligand SEMA3A is enriched in LECs compared with BECs (48), particularly in the lymphatic valves (49). Loss of SEMA3A function, either by genetic targeting or by specific blockade of its interaction with NRP1, impairs lymphatic valve leaflet elongation during development $(48,49)$. Moreover, although no NRP1 can be detected in the collecting lymphatic vessel segments between the valves (lymphangions), it is highly expressed in the valves (47). Analogous to the repulsive signaling between the SEMAs and NRPs in axon guidance, it has been suggested that the repulsion between SEMA3A and NRP1 facilitates the migration of valve leaflet cells from the vessel wall (49).

Angiopoietins and TIE2. Three angiopoietins and two TIE receptors form another system of endothelial-specific signaling (50). Loss-of-function studies of ANG1 and TIE2 in adult lymphatic vessels are not yet available; however, two gain-of-function studies indicate that ANG1 induces TIE2 phosphorylation in LECs and promotes lymphatic sprouting and growth $(51,52)$. Also, mouse ANG3 and its human cognate ANG4 promoted lymphatic sprouting and neovessel formation in a wound-healing model (53).

In contrast to the paracrine action of ANG1 produced by a variety of cells, including pericytes, SMCs, and fibroblasts, ANG2 is mainly produced by ECs and acts in an autocrine manner (54). Ang2-deleted C57BL/6 mice survive to birth without major cardiovascular defects; however, they develop lymphatic hypoplasia, chylous ascites, and peripheral edema (55). In the skin, the Ang2-deficient lymphatic vessels fail to remodel from a primitive plexus into two layers of distinct collecting vessels and capillaries (56). Consequently, the dermal lymphatic vessels provide poor fluid drainage (56). In the intestine, the growth of lacteals is arrested at the base of intestinal villi, and the mesenteric collecting lymphatic vessels lack intraluminal valves $(55,56)$. Also, TIE1 deletion was recently shown to induce defective lymphatic vasculature development (57).

The exact role of ANG2 in pathological lymphangiogenesis in adults is still unclear. In wound healing, ANG2 overexpression induces lymphangiogenesis at the wound margin (53). It is not known whether this is a direct effect of ANG2 on lymphatic vessels, since ANG2 also promotes the influx of inflammatory cells $(58,59)$, which stimulate lymphangiogenesis by secretion of growth factors and cytokines. Lymphatic-specific loss of ANG2 in cancer and other pathological conditions should answer these questions.

FGF2. Besides the more endothelial-specific factors, regulators of lymphangiogenesis include FGFs, hepatocyte growth factor, platelet-derived growth factor, insulin-like growth factors, endothelin-1, and netrin-4 $(1,60)$. However, many of these factors target a number of other cell and tissue types without specificity to the vascular system, and thus only a few of them are considered in this Review. The FGF family consists of 23 members that regulate a variety of biological functions in development, cancer, and angiogenesis (61). FGF2 induces LEC proliferation, migration, and survival as well as lymphangiogenesis in the mouse cornea, alone or in synergy with VEGFC (62-65). However, it is not yet understood whether FGF2 directly induces lymphangiogenesis or if it acts indirectly via modulation of VEGFC/VEGFR3/ VEGFR2 signaling.

In cultured LECs, FGF2 activates AKT and ERK to induce LEC proliferation, migration, and survival $(62,66)$. Loss of FGFR1 activity via gene silencing or a tyrosine kinase inhibitor abrogates the FGF2-induced LEC responses $(62,63)$. In contrast, these FGF2-induced responses were not inhibited by a VEGFR3blocking antibody or tyrosine kinase inhibitor $(62,63)$. Thus, these data suggest that FGF2 induces lymphangiogenesis directly by activating FGFR1 on LECs. However, FGF2 stimulation also increases VEGFC expression in BECs and SMCs (64). Moreover, blockade of VEGFR3 signaling inhibits FGF2-dependent corneal lymphangiogenesis $(62,64)$. These results indicate that FGF2 acts indirectly to promote lymphangiogenesis. Interestingly, LYVE1, 
Table 1

Examples of using lymphatic mechanisms for therapeutics in experimental models

\section{Disease model \\ Inhibition of pathological lymphangiogenesis \\ Corneal transplantation rejection
or suture-induced corneal inflammation
Heart transplantation rejection}

Mechanism of manipulation

Obliterative bronchiolitis

Chemical carcinogenesis in the skin

Melanoma and lung, prostate, and bladder cancer xenografts

Tumor xenografts

Neuroblastoma xenograft

Breast and gastric cancer

Orthotopic breast,

spontaneous pancreatic cancer

Lung cancer xenograft

Heterotopic brain cancer and othotopic breast cancer

Breast cancer

Pancreatic cancer xenograft

Lung cancer xenograft

Breast cancer

Breast cancer

Suture-induced inflammatory

lymphangiogenesis

Airway inflammation

HNSCC

Pancreatic cancer

Breast cancer xenograft

Melanoma

Photodynamic laser therapy

\section{Induction of therapeutic lymphangiogenesis}

Primary lymphedema

Secondary lymphedema

Secondary lymphedema

\author{
VEGFC application \\ VEGFC application \\ LN transfer and VEGFC application \\ VEGFC application
}

Hypercholesterolemia

\section{Major findings ${ }^{A}$}

Improves graft survival

Inhibits LEC-derived chemokine production and immune cell trafficking; improves allograft survival Inhibits T cell responses and obliterative bronchiolitis development

Fewer tumors and delayed onset; reduced macrophage number and inflammation

Suppresses LN and distal metastasis

Inhibits distal lymphatic dilation, SMC remodeling, and post-sentinel LN metastasis Inhibits lymphatic metastasis

Suppresses LN and distal metastasis Suppresses tumor growth and LN metastasis

Suppresses tumor growth Suppresses metastasis to LNs and lungs

A somatotropin peptide binds to NRP2 and attenuates VEGFR3 signaling

Suppresses angiogenesis, tumor growth Inhibits tumor growth and LN metastasis Inhibits tumor growth and LN metastasis

Synergistically inhibits metastasis with anti-VEGFC treatment

Suppresses inflammatory lymphangiogenesis

Reduced leukocyte influx, LV remodeling, and LN hypertrophy

Suppresses tumor lymphangiogenesis and LN metastasis; improves survival

mTOR inhibitor reduces metastasis Inhibits VEGFD-induced

prostaglandin synthesis, and thereby collecting lymphatic vessel dilation and metastasis

Destroys tumor-bearing lymphatic vessels and inhibits metastasis

Reduces primary lymphedema in Chy mice Reduces surgery-induced lymphedema in rabbits Recovery of lymphatic vessel structure and function Improves RCT
References

11,12 93

95

136

138-141

92

142

143-145

146

147

46

99

100

103

71

148

115

109

108

107

122

43

126

125, 130,

131,150

133

Aln addition to inhibition of lymphangiogenesis. HNSCC, head and neck squamous cell carcinoma; TK, tyrosine kinase.

a common lymphatic marker with elusive function, was recently found to bind FGF2 and to promote FGF2-induced signaling activation and lymphangiogenesis (67). Overall, such results could be explained if, for example, FGFR1 and VEGFR3 bind a common signal transducer in LECs.

Sphingosine 1-phosphate. Sphingosine 1-phosphate (S1P) is a bioactive lysophospholipid that regulates vascular morphogenesis, vessel tone, endothelial permeability, leukocyte adhesion, and inflammation $(68,69)$. In vitro, S1P induces LEC migration and tube formation via the S1P1/G $/ \mathrm{PLC} / \mathrm{Ca}^{2+}$ pathway $(70,71)$. Moreover, S1P stimulates ANG2 secretion from LECs much more potently than from BECs (72). Given that ANG2 is required for lymphatic development $(55,56)$, S1P may act synergistically with ANG2 in lymphangiogenesis. In vivo, S1P induces lymphangiogenesis in the Matrigel plug assay (70). S1P also can act in an autocrine manner. When Sphk1 was deleted from the mouse lym- 


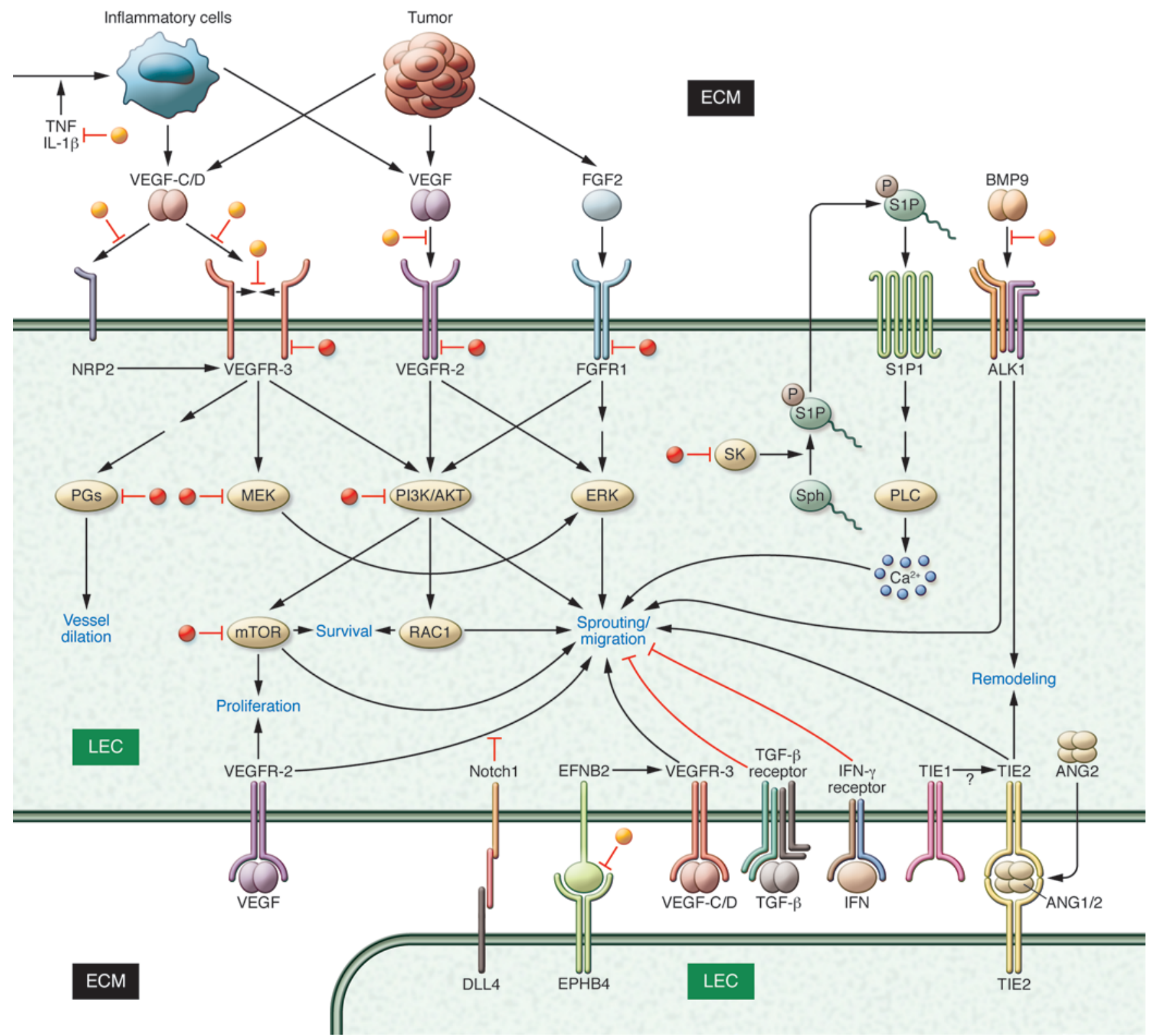

Figure 1

Growth factor and cytokine signaling pathways in lymphangiogenesis. Growth factors derived from tumor cells, inflammatory cells, or other cell types activate their receptors on the surface of LECs and initiate various signaling cascades, leading to lymphatic vessel growth. Coordinately, these factors regulate cell migration, proliferation, and survival and vessel dilation and remodeling. Interactions between adjacent LECs via ANG/ TIE2, DLL4/Notch1, and EFNB2/EPHB4 also contribute to the regulation of lymphangiogenic activity. Note that in contrast to ANG1, ANG2 is predominantly an autocrine ligand and that both angiopoietins bind with equal affinity to TIE2 and induce in trans ligand receptor complexes among ECs (103). Inhibitors that target the various involved molecules are indicated in red, and some of the key signaling consequences in blue.

phatic endothelium, S1P was absent from the lymph but not from the serum, and the lymphatic capillaries developed an irregular morphology characterized by disorganized cell-cell junctions (73). These results suggest that the LEC-derived S1P is required for normal lymphatic patterning.

$B M P 9$ and ALK1. BMP9 and its receptor, activin receptor-like 1 (ALK1), belong to the TGF- $\beta$ superfamily. In a recent study, inhibition of ALK1 with a soluble extracellular domain of the receptor or a specific blocking antibody in neonatal mice result- ed in a reduction in lymphatic capillary density and growth (74). Similarly, deletion of $B m p 9$ significantly reduced the number and maturation of lymphatic valves, leading to defective lymphatic drainage (75). Moreover, BMP9 upregulates the expression of a number of LEC genes that control lymphatic valve formation (75).

Notch1 and ephrin B2. In addition to the paracrine regulation of LECs by soluble growth factors secreted into the lymphatic vessel microenvironment, adjacent LECs undergo an intricate 


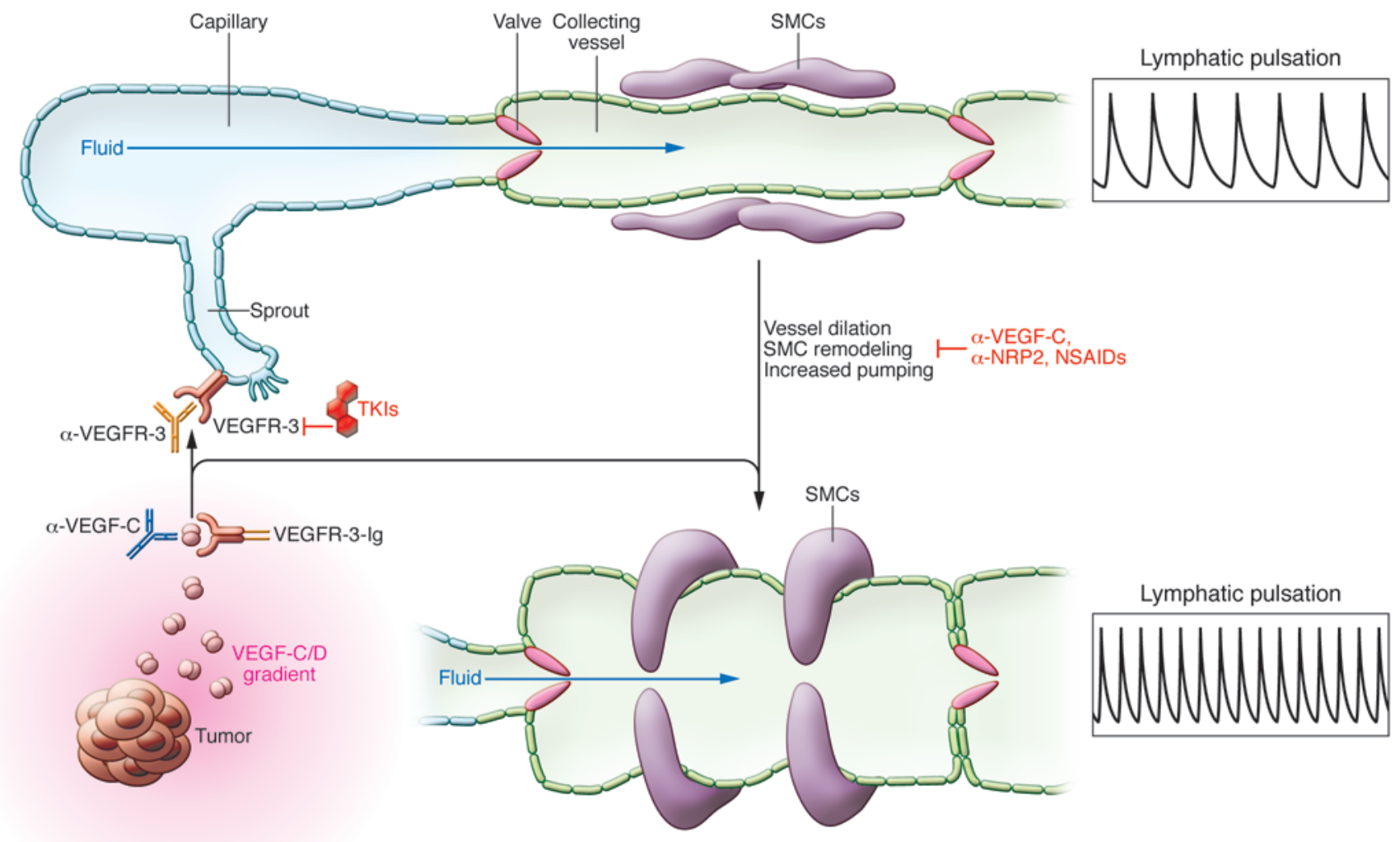

\section{Figure 2}

Inhibition of tumor-induced lymphangiogenesis and vessel remodeling. VEGFC/D is commonly produced by tumor cells and by inflammatory cells in tumors, and the resulting growth factor gradient induces the sprouting and growth of lymphatic vessels toward the tumor. Tumor-derived VEGFC/D also induces dilation, SMC remodeling, and increased pulsation of the collecting vessels. These tumor-induced processes can be attenuated by antibodies targeting the VEGFC/VEGFD/VEGFR3 signaling pathway. TKI, tyrosine kinase inhibitor.

communication among themselves via the Notch and ephrin/ Eph pathways.

Significant progress has been made during the recent decade in the establishment of the role of Notch signaling in the tip/stalk cell specification during angiogenic sprouting $(76,77)$. However, only a few studies thus far have addressed Notch signaling in lymphatic vessels. Notch1 and Notch4 are expressed by LECs in the dermis and in tumors (78). In zebrafish and mouse neonates, Notch inhibition impaired lymphatic development (79); however, in adult mice and in 3-dimensional sprouting assays of cultured LECs, Notch inhibition induced lymphatic sprouting synergistically with VEGF (36). Consistent with this observation, conditional deletion of Notch1 from the LECs during embryonic development increased LEC numbers and lymph sac size (80).

The lymphatic vessels in ephrin B2 (Efnb2) mutant mice are hypoplastic, mispatterned, and devoid of intraluminal valves, and the mutant mice develop chylothorax (81). EFNB2 in LECs facilitates VEGFR3 signaling by inducing its internalization into endosomal vesicles to maximize its signaling output (82).

Endogenous inhibitors of lymphangiogenesis. Suppressive mechanisms exist in most biological processes to maintain balance and homeostasis. TGF- $\beta$, IFN- $\gamma$, BMP2, and endostatin are examples of factors that are lymphangiogenesis suppressing. TGF- $\beta$ inhibits LEC proliferation, cord formation, and migration toward VEGFC in vitro, and also inhibits tumor- and wound healing-associated lymphangiogenesis in vivo (83-85). IFN- $\gamma$ secreted by T cells suppresses LN lymphangiogenesis through the classic JAK/STAT pathway (86). Endostatin, a proteolytic fragment of type IV collagen, can apparently inhibit tumor lymphangiogenesis by several mechanisms (87). BMP2 is induced in BECs, but not LECs, via VEGFR2 signaling (88). Normally, BMP2 signaling activity is reduced in developing LECs. However, when overexpressed, BMP2 negatively regulates the formation of LECs by downregulating PROX1 via miR-31 and miR-181a (89). It is likely that many other signals impinge on lymphangiogenesis regulation by inhibiting various necessary signal transduction events.

\section{Translation of lymphangiogenic mechanisms into therapeutics}

Although many lymphangiogenic mechanisms in both physiology and pathology remain elusive, a framework of the major signaling mechanisms can be drawn (Figure 1). An important question is how one can take advantage of these mechanisms to manipulate lymphangiogenesis for therapeutic purposes. Such attempts have already been made in numerous preclinical disease models (Table 1), including cancer (Figure 2) and lymphedema (Figure 3).

Inhibition of pathological lymphangiogenesis. Lymphangiogenic signaling is highly active during development and decreases to very 


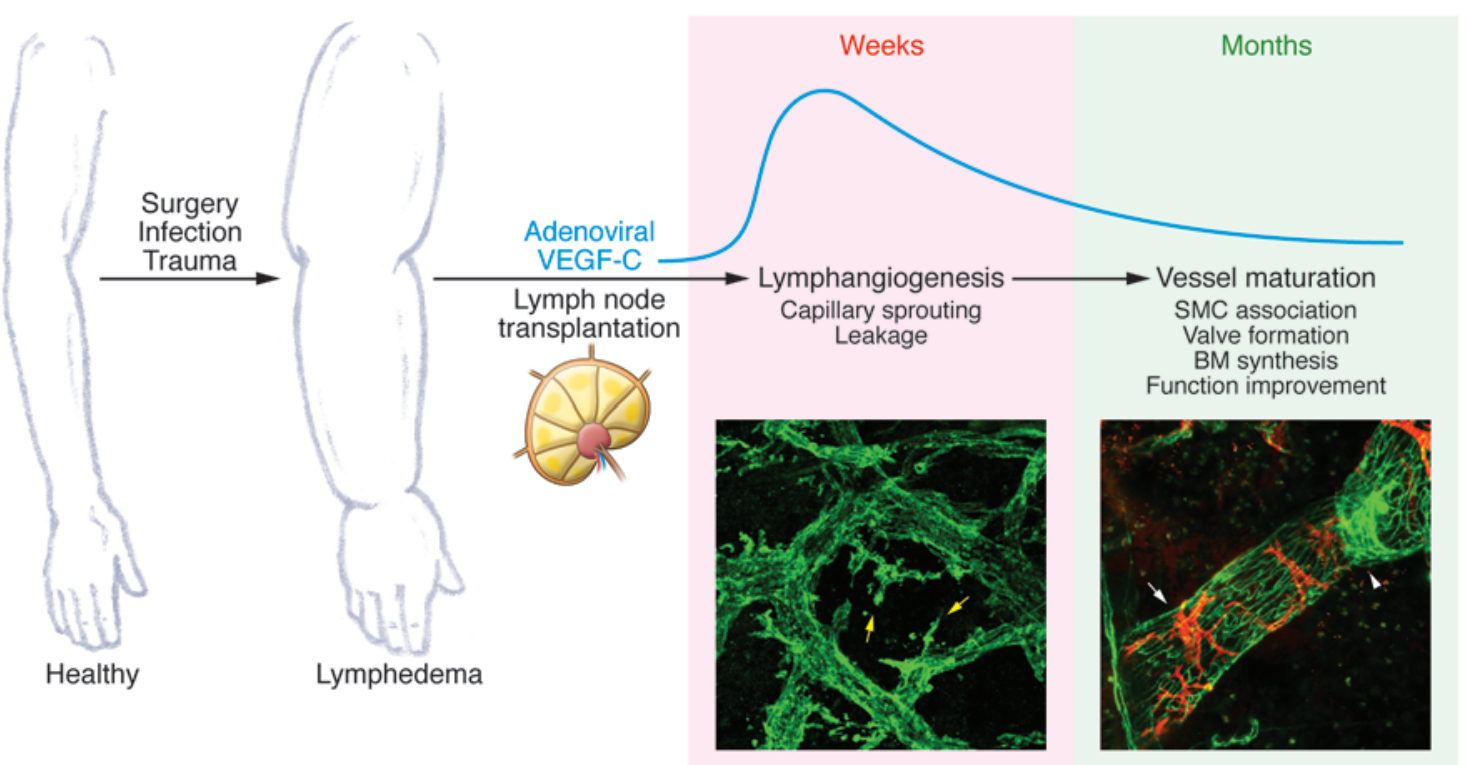

\section{Figure 3}

VEGFC-induced regeneration of lymphatic vessels for the treatment of lymphedema. Surgery, infection, or trauma damages the lymphatic vessels, leading to secondary lymphedema. Adenoviral VEGFC treatment with or without LN transplantation may be used to restore functional lymphatic vessels at the damaged site. Adenoviral VEGFC expression reaches peak levels soon after injection and lasts for about two to three weeks. The newly generated lymphatic vessels undergo intrinsic remodeling processes, finally maturing into collecting vessels with associated SMCs and intraluminal valves within six months in mice and pigs $(125,131)$. The blue curve indicates the quantitative dynamics of VEGFC expression. Original magnification, $\times 40$.

low levels in adulthood; however, these pathways can be re-activated in pathological conditions (90). Aberrant growth or activation of lymphatic vessels is associated with cancer and some chronic inflammatory diseases (91). In cancer, tumor-induced lymphangiogenesis facilitates lymphatic metastasis, and in allogeneic tissue transplants, inflammatory lymphangiogenesis contributes to fullblown immune responses to the grafts.

Direct inhibition of VEGFR3 signaling. Since VEGFC/VEGFR3 signaling is the most potent and specific lymphangiogenic pathway, it has attracted the most attention and effort as a target for drug development (Table 1; ref. 91). VEGFR3 signaling can be directly inhibited at several levels: by trapping the ligands with sVEGFRs, blocking ligand-receptor binding or receptor dimerization with antibodies, and by inhibiting the VEGFR3 tyrosine kinase (Figure 1). Inhibition of VEGFR3 signaling suppresses tumor lymphangiogenesis and LN metastasis in a variety of tumor models (Table 1). Interestingly, tumors also induce dilation of distal lymphatic vessels, remodeling of the associated SMCs, and frequency modulation of their pumping activity; these disease-associated changes were reversed by VEGFC or NRP2 blockade (92).

Similar inhibitors may prove useful in tissue transplantation, as inhibition of VEGFR3 signaling suppresses tissue allograft-induced lymphangiogenesis and improves graft survival in mouse models $(11,12,93-95)$. Notably, the neo-lymphatic vessels induced in tumors or allografts are not only passive conduits; they also secrete chemokines such as CCL21 to attract tumor cells or immune cells (95-98).

Besides blocking ligand-receptor binding, prevention of receptor dimerization and subsequent receptor transactivation is another effective approach that provides additive inhibition when used with antibodies that block ligand binding (17). Future stud- ies will determine whether such strategies can be translated into effective therapeutics.

Targeting VEGFR3 coreceptors. VEGFR3 signaling can also be attenuated by targeting its coreceptors. Specific inhibition of VEGFC binding to NRP2 with a blocking antibody has been shown to suppress tumor lymphangiogenesis and metastasis to sentinel LNs and lungs (46). Binding of NRP2 by a somatotropin peptide inhibits VEGFR2/VEGFR3/NRP1/NRP2 complex formation, leading to reduced tumor lymphangiogenesis (99). Furthermore, EFNB2 is required for full VEGFR3 activity (82) and a specific blocking antibody inhibiting the EFNB2/EPHB4 interaction suppresses tumor lymphangiogenesis (100).

Blocking other lymphangiogenic factors. ANG2-blocking antibodies have been shown to inhibit tumor angiogenesis and growth $(101,102)$. Recently, these antibodies were also shown to inhibit tumor lymphangiogenesis and LN metastasis, presumably via an effect on EC-cell junctions (103). Furthermore, S1P levels are elevated in breast cancer patients, and in a mouse model of breast cancer, a SPHK1 inhibitor reduced tumor angiogenesis and lymphangiogenesis and decreased the overall tumor burden as well as LN and lung metastasis (71). Notch pathway inhibition with $\gamma$-secretase inhibitors and anti-DLL4 antibodies leads to excessive yet nonproductive angiogenesis, which inhibits tumor growth $(104,105)$. Notch inhibition also induces lymphatic sprouting and outgrowth $(36,80)$. Future studies will determine whether Notch inhibition similarly leads to nonproductive lymphangiogenesis and reduced lymphatic metastasis.

Inhibition by indirect mechanisms. Future therapeutic strategies might also be based on indirect downregulation of VEGFC expression in the microenvironment of lymphatic vessels. For example, 
knockdown or inhibition of FGFR2 (106) or mTOR $(107,108)$ in tumor cells inhibits VEGFC production, leading to reduced tumor lymphangiogenesis and metastasis. However, such strategies might suffer from lack of specificity.

Inflammatory cytokines, such as TNF- $\alpha$ (109) and IL-1 $\alpha$ (110), stimulate lymphangiogenesis by recruiting VEGFC/D-secreting inflammatory cells $(111,112)$. Endotoxin also induces lymphangiogenesis indirectly by stimulating LECs to secrete chemokines that attract VEGFC/D-producing macrophages (113). Inhibition of inflammatory cell infiltration or inflammatory signaling pathways in general reduces the supply of lymphangiogenic factors. TNF- $\alpha$-blocking antibodies and deletion of TNF receptor diminish leukocyte influx during airway infection and the associated lymphatic vessel remodeling and LN hypertrophy (109). Systemic or local depletion of macrophages alleviates sutureinduced inflammatory lymphangiogenesis in the cornea $(30,114)$. In particular, VEGFC/D production by $\mathrm{CD} 36^{+}$macrophages is inhibited by endogenous thrombospondin-1, and treatment with recombinant thrombospondin-1 suppresses corneal lymphangiogenesis (115). A cyclooxygenase-2 inhibitor also suppresses lymphangiogenesis, presumably by inhibiting VEGFC/D production from prostaglandin-activated macrophages (116). Furthermore, anti-VEGF treatment also limits inflammatory lymphangiogenesis $(117,118)$. In addition to its possible direct anti-lymphangiogenic effects, anti-VEGF can block the recruitment of VEGFR1expressing inflammatory cells (30-32), and its anti-angiogenic activity may reduce the influx of inflammatory cells in general.

Inflammation-associated lymphangiogenesis is beneficial in some cases. Blockade of lymphangiogenesis causes bronchial lymphedema and exacerbates airflow obstruction (119) and worsens the edema induced by delayed-type hypersensitivity $(117,120)$. Moreover, it delays antigen clearance and inflammation resolution (114). In contrast, enhancement of inflammation-induced lymphangiogenesis by VEGFC overexpression alleviates edema $(114,117,120)$. Nevertheless, caution is needed when inducing lymphangiogenesis for facilitation of inflammation resolution, as this might, in theory, also increase the drainage of unfiltered pathogens and inflammatory mediators through the lymphatic vasculature (121).

Targeting lymphangiogenic signaling pathways downstream of VEGFR3. Signaling molecules and effectors downstream of the lymphatic endothelial growth factor receptors are also targetable. VEGFD signaling, but not VEGFC signaling, suppresses expression of a prostaglandin-degrading enzyme in the collecting lymphatic vessels (122). The resulting accumulation of prostaglandins induces dilation of the collecting vessels and facilitates cancer metastasis, a process that can be inhibited by prostaglandin inhibitors (122). Several lymphangiogenic factors activate mTOR in LECs, and mTOR inhibition blocks lymphangiogenesis (66). In an apparent positive feedback loop, mTOR inhibition reduces VEGFR3 protein level by promoting its degradation in LECs, thereby decreasing their responsiveness to lymphangiogenic factors (123). MEK1/2 and Rac1 have emerged as important signaling molecules downstream of VEGF/S1P/FGF2 in a screen for drugs capable of inhibiting lymphatic sprouting (124). Since many of the drugs used in the screen are available or are in clinical trials for cancer therapy, it would be interesting to test whether they also have anti-lymphangiogenic activities.

Therapeutic lymphangiogenesis and recovery of lymphatic function. Lymphedema is characterized by the impairment of lymphatic transport capacity due to abnormal vessel development or destruc- tion. Currently, lymphedema is a life-long disease without effective treatment. Lymphangiogenic growth factor therapy may become the most appropriate form of curative treatment for most secondary lymphedemas (Figure 3) (91).

Unprocessed forms of VEGFC and VEGFD signal preferentially through VEGFR3 (7). VEGFC gene therapy can restore a functional network of lymphatic vessels and reduction in edema in primary (43) and secondary lymphedema mouse models $(125,126)$. While full-length VEGFC and VEGFD predominantly induced lymphangiogenesis, the VEGFR3-selective mutant form of VEGFC $\left(\operatorname{VEGFC}^{1565}\right)(7,127)$ induces only lymphangiogenesis in skeletal muscle and skin $(128,129)$. Furthermore, normal lymphatic function is restored when the $\mathrm{K} 14-\mathrm{VEGFC}{ }^{156 \mathrm{~S}}$ transgene is introduced into Chy mice that suffer from hereditary lymphedema (43).

Transient overexpression of VEGFC promotes lymphatic capillary growth, followed by long-term lymphatic vessel remodeling, differentiation, and maturation, resulting in the formation of a functional network of collecting lymphatic vessels with valves and smooth muscle cell coverage (125). When VEGFC gene therapy was combined with LN transplantation in a mouse model of postmastectomy lymphedema, the functional outcome was enhanced. Restoration of functional afferent and efferent lymphatic vessels was obtained, which prevented the regression of the transplanted LNs (125). Similar strategies have been used to repair damaged lymphatic vessels in a large animal model (130). In pigs, both VEGFC and VEGFD gene therapy induced robust growth of lymphatic vessels in a surgically LN-evacuated groin area, causing a significant improvement in postsurgical lymphatic drainage (131).

Obesity, diabetes, and atherosclerosis raise significant health concerns of increasing importance. Obesity is associated with impaired lymphatic vessel function in humans and mice $(132,133)$. Lymphatic vessels have been recently identified as a critical regulator of reverse cholesterol transport (RCT), which removes excess cholesterol from peripheral tissues $(133,134)$. RCT was severely impaired when lymphangiogenesis was blocked in mice receiving transplanted atherosclerotic aortas, or when lymphatic drainage in peripheral tissues was surgically obstructed $(133,134)$. In contrast, restoration of lymphatic drainage by VEGFC treatment in hypercholesterolemic mice improved RCT (133). Thus, enhancement of lymphatic function might represent a new therapeutic approach to prevent cholesterol accumulation in diseases such as atherosclerosis, diabetes, and dyslipidemia.

\section{Conclusions and future perspectives}

Agents that target lymphangiogenesis may also affect blood vessels, inflammatory cells, tumor-associated macrophages, and tumor cells. Thus, reduced lymphangiogenesis in a tumor or in inflamed tissue may be secondary to, for example, reduction in VEGFC secretion or infiltration of growth factor-producing inflammatory cells. While it is important to distinguish specific effects from indirect or concomitant side effects for basic research, it may be less crucial for disease outcome, as lymphangiogenesis, angiogenesis, and neoplasia share many pathways and mechanisms. Targeting the common components of these processes may yield additional benefits; however, the benefits of multi-targeting should be weighed against the risk of side effects. Further investigations are required to determine the best strategy for lymphangiogenesis inhibition in each case. When promoting lymphangiogenesis, the approach should be specific to lymphatic vessels in most cases, employing factors with minimal angiogenic or potentially deleterious activities. 
Further research is needed to solve the many basic and translational questions that remain. The functions of factors such as angiopoietins and FGFs in lymphangiogenesis require analysis that is best done in genetic mouse models. How do the intracellular signaling pathways of these factors differ between the BECs and LECs? What keeps blood and lymphatic vessels apart from each other after their separation in embryos? Which immune functions are dependent on lymphatic vessels, and can one treat inflammatory diseases by manipulating lymphatic vessels? What regulates the persistence of lymphatic vessels generated in pathological conditions or in response to therapy? Does lymph flow play other functions in vessel homeostasis besides valve formation (135)? How do the responses of collecting lymphatic vessels and lymphatic capillaries to VEGFC stimulation differ? What is the best strategy to deliver VEGFC or VEGFC plus CCBE1 for the treatment of lymphedema? Do the coreceptors or CCBE1 have VEGFR3-independent activities in lymphangiogenesis? Can one manipulate lymphatic vessels to regulate fat transport or to counteract cholesterol deposition in tissues?

Current knowledge of lymphangiogenic mechanisms has already provided useful strategies and yielded promising results in many preclinical studies. These now await further validation by clinical trials. Further understanding of the mechanisms that emerge from the basic research and translational studies should provide guidance for the rational design of disease treatments directed to lymphatic vessels.

\section{Acknowledgments}

The studies in our research group are currently supported by the Academy of Finland, the European Research Council (grant ERC2010-AdG-268804), the Sigrid Juselius Foundation, the Leducq Foundation (grant 11CVD03), and the Finnish Cancer Organizations. We apologize to the many authors whose work could not be cited due to the citation limit and potential overlap with the other Reviews published in this same issue.

Address correspondence to: Kari Alitalo, Wihuri Research Institute and Translational Cancer Biology Program, P.O.B. 63 (Haartmaninkatu 8), FIN-00014, University of Helsinki, Finland. Phone: 358.9.1912.5511; Fax: 358.9.1912.5510; E-mail: kari.alitalo@helsinki.fi.
1. Tammela T, Alitalo K. Lymphangiogenesis: Molecular mechanisms and future promise. Cell. 2010; 140(4):460-476.

2. Koltowska K, Betterman KL, Harvey NL, Hogan BM. Getting out and about: the emergence and morphogenesis of the vertebrate lymphatic vasculature. Development. 2013;140(9):1857-1870.

3. Karkkainen MJ, et al. Vascular endothelial growth factor $\mathrm{C}$ is required for sprouting of the first lymphatic vessels from embryonic veins. Nat Immunol. 2004;5(1):74-80.

4. D'Amico G, Alitalo K. Inside bloody lymphatics. Blood. 2010;116(4):512-513.

5. Schulte-Merker S, Sabine A, Petrova TV. Lymphatic vascular morphogenesis in development, physiology, and disease. J Cell Biol. 2011;193(4):607-618.

6. Jeltsch M, et al. Hyperplasia of lymphatic vessels in VEGF-C transgenic mice. Science. 1997; 276(5317):1423-1425.

7. Joukov V, et al. A novel vascular endothelial growth factor, VEGF-C, is a ligand for the Flt4 (VEGFR-3) and KDR (VEGFR-2) receptor tyrosine kinases. EMBO J. 1996;15(7):1751-298.

8. Kaipainen A, et al. Expression of the fms-like tyrosine kinase 4 gene becomes restricted to lymphatic endothelium during development. Proc Natl Acad SciU S A. 1995;92(8):3566-3570.

9. Hägerling R, et al. A novel multistep mechanism for initial lymphangiogenesis in mouse embryos based on ultramicroscopy. EMBO J. 2013;32(5):629-644.

10. Mäkinen T, et al. Inhibition of lymphangiogenesis with resulting lymphedema in transgenic mice expressing soluble VEGF receptor-3. Nat Med. 2001; 7(2):199-205.

11. Albuquerque RJC, et al. Alternatively spliced vascular endothelial growth factor receptor- 2 is an essential endogenous inhibitor of lymphatic vessel growth. Nat Med. 2009;15(9):1023-1030.

12. Singh N, et al. Soluble vascular endothelial growth factor receptor 3 is essential for corneal alymphaticity. Blood. 2013;121(20):4242-4249.

13. Lemmon MA, Schlessinger J. Cell signaling by receptor tyrosine kinases. Cell. 2010;141(7):1117-1134.

14. Scott JD, Pawson T. Cell Signaling in space and time: where proteins come together and when they're apart. Science. 2009;326(5957):1220-1224.

15. Mäkinen T, et al. Isolated lymphatic endothelial cells transduce growth, survival and migratory signals via the VEGF-C/D receptor VEGFR-3. EMBOJ. 2001;20(17):4762-4773.

16. Coso S, Zeng Y, Opeskin K, Williams ED. Vascular endothelial growth factor receptor-3 directly interacts with phosphatidylinositol 3-kinase to regulate lymphangiogenesis. PLoS One. 2012;7(6):e39558.

17. Tvorogov D, et al. Effective suppression of vascular network formation by combination of antibodies blocking VEGFR ligand binding and receptor dimerization. Cancer Cell. 2010;18(6):630-640.

18. Mouta-Bellum C, et al. Organ-specific lymphangiectasia, arrested lymphatic sprouting, and maturation defects resulting from gene-targeting of the PI3K regulatory isoforms $\mathrm{p} 85 \alpha, \mathrm{p} 55 \alpha$, and $\mathrm{p} 50 \alpha$. Dev Dyn. 2009;238(10):2670-2679.

19. Gupta S, et al. Binding of ras to phosphoinositide 3-kinase p110alpha is required for ras-driven tumorigenesis in mice. Cell. 2007;129(5):957-968.

20. D'Amico G, et al. Regulation of lymphatic-blood vessel separation by endothelial Rac1. Development. 2009;136(23):4043-4053.

21. Dixelius J, et al. Ligand-induced vascular endothelial growth factor receptor-3 (VEGFR-3) heterodimerization with VEGFR-2 in primary lymphatic endothelial cells regulates tyrosine phosphorylation sites. J Biol Chem. 2003;278(42):40973-40979.

22. Karkkainen MJ, et al. Missense mutations interfere with VEGFR-3 signalling in primary lymphoedema. Nat Genet. 2000;25(2):153-159.

23. Gordon K, et al. FLT4/VEGFR3and Milroy disease: novel mutations, a review of published variants and database update. Hum Mutat. 2012;34(1):23-31.

24. Ghalamkarpour A, et al. Recessive primary congenital lymphoedema caused by a VEGFR3 mutation. J Med Genet. 2009;46(6):399-404.

25. Ghalamkarpour A, et al. Hereditary lymphedema type I associated with VEGFR3 mutation: the first de novo case and atypical presentations. Clin Genet. 2006;70(4):330-335.

26. Gordon K, et al. Mutation in vascular endothelial growth factor-C, a ligand for vascular endothelial growth factor receptor-3, is associated with autosomal dominant milroy-like primary lymphedema. Circ Res. 2013;112(6):956-960.

27. Wirzenius $M$, et al. Distinct vascular endothelial growth factor signals for lymphatic vessel enlargement and sprouting. J Exp Med. 2007; 204(6):1431-1440.

28. Bjorndahl MA, et al. Vascular endothelial growth factor-a promotes peritumoral lymphangiogenesis and lymphatic metastasis. Cancer Res. 2005; 65(20):9261-9268.

29. Hirakawa S, et al. VEGF-A induces tumor and sentinel lymph node lymphangiogenesis and promotes lymphatic metastasis. J Exp Med. 2005; 201(7):1089-1099.

30. Cursiefen C, et al. VEGF-A stimulates lymphangiogenesis and hemangiogenesis in inflammatory neovascularization via macrophage recruitment. J Clin Invest. 2004;113(7):1040-1050.

31. Kerber M, et al. Flt-1 signaling in macrophages promotes glioma growth in vivo. Cancer Res. 2008; 68(18):7342-7351.

32. Murakami M, et al. VEGFR1 tyrosine kinase signaling promotes lymphangiogenesis as well as angiogenesis indirectly via macrophage recruitment. Arterioscler Thromb Vasc Biol. 2008;28(4):658-664.

33. Nakao S, et al. Blood vessel endothelial VEGFR-2 delays lymphangiogenesis: an endogenous trapping mechanism links lymph- and angiogenesis. Blood. 2011;117(3):1081-1090.

34. Benest AV, Harper SJ, Herttuala SY, Alitalo K, Bates DO. VEGF-C induced angiogenesis preferentially occurs at a distance from lymphangiogenesis. Cardiovasc Res. 2008;78(2):315-323.

35. Dellinger MT, Meadows SM, Wynne K, Cleaver O, Brekken RA. Vascular endothelial growth factor receptor-2 promotes the development of the lymphatic vasculature. PLoS One. 2013;8(9):e74686.

36. Zheng W, et al. Notch restricts lymphatic vessel sprouting induced by vascular endothelial growth factor. Blood. 2011;118(4):1154-1162.

37. Hogan BM, et al. Ccbe 1 is required for embryonic lymphangiogenesis and venous sprouting. Nat Genet. 2009;41(4):396-398.

38. Bos FL, et al. CCBE1 is essential for mammalian lymphatic vascular development and enhances the lymphangiogenic effect of vascular endothelial growth factor-C in vivo. Circ Res. 2011;109(5):486-491.

39. Alders $M$, et al. Mutations in CCBE1 cause generalized lymph vessel dysplasia in humans. Nat Genet. 2009;41(12):1272-1274.

40. Muratori C, Tamagnone L. Semaphorin signals tweaking the tumor microenvironment. Adv Cancer Res. 2012;114:59-85.

41. Sakurai A, Doci C, Gutkind JS. Semaphorin signaling in angiogenesis, lymphangiogenesis and cancer. Cell Res. 2011;22(1):23-32.

42. Herzog Y, Kalcheim C, Kahane N, Reshef R, Neufeld G. Differential expression of neuropilin-1 and neuropilin-2 in arteries and veins. Mech Dev. 2001; 109(1):115-119.

43. Karkkainen MJ, et al. A model for gene therapy of human hereditary lymphedema. Proc Natl Acad Sci US A. 2001;98(22):12677-12682. 
44. Yuan L, et al. Abnormal lymphatic vessel development in neuropilin 2 mutant mice. Development. 2002;129(20):4797-4806

45. Karpanen T, et al. Functional interaction of VEGF$\mathrm{C}$ and VEGF-D with neuropilin receptors. FASEBJ. 2006;20(9):1462-1472.

46. Caunt $M$, et al. Blocking neuropilin-2 function inhibits tumor cell metastasis. Cancer Cell. 2008;13(4):331-342.

47. Xu Y, et al. Neuropilin-2 mediates VEGF-C-induced lymphatic sprouting together with VEGFR3. J Cell Biol. 2010;188(1):115-130.

48. Jurisic G, et al. An unexpected role of semaphorin3a-neuropilin-1 signaling in lymphatic vessel maturation and valve formation. Circ Res. 2012; 111(4):426-436.

49. Bouvrée K, et al. Semaphorin3A, Neuropilin-1, and PlexinA1 are required for lymphatic valve formation. Circ Res. 2012;111(4):437-445.

50. Augustin HG, Koh GY, Thurston G, Alitalo K. Control of vascular morphogenesis and homeostasis through the angiopoietin-Tie system. Nat Rev Mol Cell Biol. 2009;10(3):165-177.

51. Tammela T, et al. Angiopoietin-1 promotes lymphatic sprouting and hyperplasia. Blood. 2005; 105(12):4642-4648.

52. Morisada T, et al. Angiopoietin-1 promotes LYVE1-positive lymphatic vessel formation. Blood. 2005; 105(12):4649-4656.

53. Kim KE, et al. In vivo actions of angiopoietins on quiescent and remodeling blood and lymphatic vessels in mouse airways and skin. Arterioscler Thromb Vasc Biol. 2007;27(3):564-570.

54. Daly C, et al. Angiopoietin-2 functions as an autocrine protective factor in stressed endothelial cells. Proc Natl Acad Sci US A. 2006;103(42):15491-15496.

55. Gale NW, et al. Angiopoietin-2 is required for postnatal angiogenesis and lymphatic patterning, and only the latter role is rescued by Angiopoietin-1. Dev Cell. 2002;3(3):411-423.

56. Dellinger $M$, et al. Defective remodeling and maturation of the lymphatic vasculature in Angiopoietin-2 deficient mice. Dev Biol. 2008;319(2):309-320.

57. D'Amico G, et al. Tie1 deletion inhibits tumor growth and improves angiopoietin antagonist therapy. J Clin Invest. 2014;124(2):824-834.

58. Fiedler $U$, et al. Angiopoietin-2 sensitizes endothelial cells to TNF- $\alpha$ and has a crucial role in the induction of inflammation. Nat Med. 2006 12(2):235-239.

59. Tabruyn SP, et al. Angiopoietin-2-driven vascular remodeling in airway inflammation. Am J Pathol. 2010;177(6):3233-3243.

60. Larrieu-Lahargue F, Welm AL, Thomas KR, Li DY Netrin-4 induces lymphangiogenesis in vivo. Blood 2010;115(26):5418-5426

61. Turner N, Grose R. Fibroblast growth factor signalling: from development to cancer. Nat Rev Cancer. 2010;10(2):116-129.

62. Cao R, et al. Collaborative interplay between FGF-2 and VEGF-C promotes lymphangiogenesis and metastasis. Proc Natl Acad Sci U S A. 2012; 109(39):15894-15899.

63. Kazenwadel J, Secker GA, Betterman KL, Harvey NL. In vitro assays using primary embryonic mouse lymphatic endothelial cells uncover key roles for FGFR1 signalling in lymphangiogenesis. PLoS One. 2012;7(7):e40497.

64. Kubo H, et al. Blockade of vascular endothelial growth factor receptor-3 signaling inhibits fibroblast growth factor-2-induced lymphangiogenesis in mouse cornea. Proc Natl Acad Sci U S A. 2002;99(13):8868-8873.

65. Chang LK, et al. Dose-dependent response of FGF-2 for lymphangiogenesis. Proc Natl Acad Sci U S A. 2004;101(32):11658-11663

66. Matsuo M, Yamada S, Koizumi K, Sakurai H, Saiki I. Tumour-derived fibroblast growth factor- 2 exerts lymphangiogenic effects through $\mathrm{Akt} / \mathrm{mTOR} /$
p70S6kinase pathway in rat lymphatic endothelial cells. Eur J Cancer. 2007;43(11):1748-1754

67. Platonova $\mathrm{N}$, et al. Evidence for the interaction of fibroblast growth factor- 2 with the lymphatic endothelial cell marker LYVE-1. Blood. 2013; 121(7):1229-1237.

68. Lucke S, Levkau B. Endothelial functions of sphingosine-1-phosphate. Cell Physiol Biochem. 2010; 26(1):87-96.

69. Mochizuki N. Vascular integrity mediated by vascular endothelial cadherin and regulated by sphingosine 1-phosphate and angiopoietin-1. Circ J. 2009;73(12):2183-2191.

70. Yoon CM, et al. Sphingosine-1-phosphate promotes lymphangiogenesis by stimulating S1P1/ Gi/PLC/Ca2+ signaling pathways. Blood. 2008; 112(4):1129-1138.

71. Nagahashi M, et al. Sphingosine-1-phosphate produced by sphingosine kinase 1 promotes breast cancer progression by stimulating angiogenesis and lymphangiogenesis. Cancer Res. 2012;72(3):726-735.

72. Jang C, et al. Angiopoietin-2 exocytosis is stimulated by sphingosine-1-phosphate in human blood and lymphatic endothelial cells. Arterioscler Thromb Vasc Biol. 2009;29(3):401-407.

73. Pham THM, et al. Lymphatic endothelial cell sphingosine kinase activity is required for lymphocyte egress and lymphatic patterning. J Exp Med. 2010; 207(1):17-27.

74. Niessen K, Zhang G, Ridgway JB, Chen H, Yan M. ALK1 signaling regulates early postnatal lymphatic vessel development. Blood. 2010;115(8):1654-1661.

75. Levet $\mathrm{S}$, et al. Bone morphogenetic protein 9 (BMP9) controls lymphatic vessel maturation and valve formation. Blood. 2013;122(4):598-607.

76. Blanco R, Gerhardt H. VEGF and Notch in tip and stalk cell selection. Cold Spring Harb Perspect Med. 2013;3(1):a006569.

77. Benedito R, Hellström M. Notch as a hub for signaling in angiogenesis. Exp Cell Res. 2013; 319(9):1281-1288.

78. Shawber CJ, et al. Notch alters VEGF responsiveness in human and murine endothelial cells by direct regulation of VEGFR-3 expression. J Clin Invest. 2007;117(11):3369-3382.

79. Geudens I, et al. Role of delta-like-4/Notch in the formation and wiring of the lymphatic network in zebrafish. Arterioscler Thromb Vasc Biol. 2010; 30(9):1695-1702

80. Murtomäki A, et al. Notch1 functions as a negative regulator of lymphatic endothelial cell differentiation in the venous endothelium. Development. 2013;140(11):2365-2376

81. Mäkinen T, et al. PDZ interaction site in ephrinB2 is required for the remodeling of lymphatic vasculature. Genes Dev. 2005;19(3):397-410.

82. Wang Y, et al. Ephrin-B2 controls VEGF-induced angiogenesis and lymphangiogenesis. Nature. 2010;465(7297):483-486

83. Oka M, et al. Inhibition of endogenous TGF- $\beta$ signaling enhances lymphangiogenesis. Blood. 2008; 111(9):4571-4579.

84. Clavin NW, et al. TGF-beta1 is a negative regulator of lymphatic regeneration during wound repair. $A m J$ Physiol Heart Circ Physiol. 2008;295(5):H2113-H2127.

85. Avraham T, et al. Blockade of transforming growth factor- $\beta 1$ accelerates lymphatic regeneration during wound repair. Am J Pathol. 2010;177(6):3202-3214.

86. Kataru RP, et al. T lymphocytes negatively regulate lymph node lymphatic vessel formation. Immunity. 2011;34(1):96-107.

87. Brideau $\mathrm{G}$, et al. Endostatin overexpression inhibits lymphangiogenesis and lymph node metastasis in mice. Cancer Res. 2007;67(24):11528-11535.

88. Veikkola T, et al. Intrinsic versus microenvironmental regulation of lymphatic endothelial cell phenotype and function. FASEB J. 2003;17(14):2006-2013. 89. Dunworth WP, et al. Bone morphogenetic pro- tein 2 signaling negatively modulates lymphatic development in vertebrate embryos. Circ Res. 2014; 114(1):56-66

90. Martínez-Corral I, et al. In vivo imaging of lymphatic vessels in development, wound healing, inflammation, and tumor metastasis. Proc Natl Acad Sci U S A. 2012;109(16):6223-6228.

91. Alitalo $\mathrm{K}$. The lymphatic vasculature in disease. Nat Med. 2011;17(11):1371-1380.

92. Gogineni A, et al. Inhibition of VEGF-C modulates distal lymphatic remodeling and secondary metastasis. PLoS One. 2013;8(7):e68755.

93. Uehara H, et al. Dual suppression of hemangiogenesis and lymphangiogenesis by splice-shifting morpholinos targeting vascular endothelial growth factor receptor 2 (KDR). FASEBJ. 2013;27(1):76-85.

94. Bock F, et al. Novel anti(lymph)angiogenic treatment strategies for corneal and ocular surface diseases. Prog Retin Eye Res. 2013;34:89-124.

95. Nykänen AI, et al. Targeting lymphatic vessel activation and CCL2 1 production by vascular endothelial growth factor receptor-3 inhibition has novel immunomodulatory and antiarteriosclerotic effects in cardiac allografts. Circulation. 2010; 121(12):1413-1422.

96. Kerjaschki D, et al. Lymphatic neoangiogenesis in human kidney transplants is associated with immunologically active lymphocytic infiltrates. J Am Soc Nephrol. 2004;15(3):603-612.

97. Issa A, Le TX, Shoushtari AN, Shields JD, Swartz MA. Vascular endothelial growth factor-C and $\mathrm{C}$-C chemokine receptor 7 in tumor cell-lymphatic cross-talk promote invasive phenotype. Cancer Res. 2009;69(1):349-357.

98. Kim M, et al. CXCR4 signaling regulates metastasis of chemoresistant melanoma cells by a lymphatic metastatic niche. Cancer Res. 2010; 70(24):10411-10421.

99. Lee E, Koskimaki JE, Pandey NB, Popel AS. Inhibition of lymphangiogenesis and angiogenesis in breast tumor xenografts and lymph nodes by a peptide derived from transmembrane protein 45A. Neoplasia. 2013;15(2):112-124.

100.Abéngozar MA, et al. Blocking ephrinB2 with highly specific antibodies inhibits angiogenesis, lymphangiogenesis, and tumor growth. Blood. 2012;119(19):4565-4576.

101. Mazzieri R, et al. Targeting the ANG2/TIE2 axis inhibits tumor growth and metastasis by impairing angiogenesis and disabling rebounds of proangiogenic myeloid cells. Cancer Cell. 2011;19(4):512-526.

102. Leow CC, et al. MEDI3617, a human antiangiopoietin 2 monoclonal antibody, inhibits angiogenesis and tumor growth in human tumor xenograft models. Int J Oncol. 2012;40(5):1321-1330.

103. Holopainen T, et al. Effects of Angiopoietin2-blocking antibody on endothelial cell-cell junctions and lung metastasis. J Natl Cancer Inst. 2012; 104(6):461-475

104. Ridgway J, et al. Inhibition of Dll4 signalling inhibits tumour growth by deregulating angiogenesis. Nature. 2006;444(7122):1083-1087.

105. Noguera-Troise I, et al. Blockade of Dll4 inhibits tumour growth by promoting non-productive angiogenesis. Nature. 2006;444(7122):1032-1037.

106. Larrieu-Lahargue $\mathrm{F}$, et al. Blocking fibroblast growth factor receptor signaling inhibits tumor growth, lymphangiogenesis, and metastasis. PLoS One. 2012;7(6):e39540.

107. Kobayashi S, et al. Rapamycin, a specific inhibitor of the mammalian target of rapamycin, suppresses lymphangiogenesis and lymphatic metastasis. Cancer Sci. 2007;98(5):726-733.

108. Patel V, et al. Decreased lymphangiogenesis and lymph node metastasis by mTOR inhibition in head and neck cancer. Cancer Res. 2011;71(22):7103-7112.

109 . Baluk P, et al. TNF- $\alpha$ drives remodeling of blood vessels and lymphatics in sustained airway inflamma- 
tion in mice. J Clin Invest. 2009;119(10):2954-2964.

110.Baluk P, et al. Transgenic overexpression of interleukin- $1 \beta$ induces persistent lymphangiogenesis but not angiogenesis in mouse airways. Am J Pathol. 2013;182(4):1434-1447.

111. Tan KW, et al. Neutrophils contribute to inflammatory lymphangiogenesis by increasing VEGF-A bioavailability and secreting VEGF-D. Blood. 2013; 122(22):3666-3677.

112. Ristimäki A, Narko K, Enholm B, Joukov V, Alitalo K. Proinflammatory cytokines regulate expression of the lymphatic endothelial mitogen vascular endothelial growth factor-C. J Biol Chem. 1998; 273(14):8413-8418.

113.Kang S, et al. Toll-like receptor 4 in lymphatic endothelial cells contributes to LPS-induced lymphangiogenesis by chemotactic recruitment of macrophages. Blood. 2009;113(11):2605-2613.

114.Kataru RP, et al. Critical role of CD $11 b+$ macrophages and VEGF in inflammatory lymphangiogenesis, antigen clearance, and inflammation resolution. Blood. 2009;113(22):5650-5659.

115. Cursiefen C, et al. Thrombospondin 1 inhibits inflammatory lymphangiogenesis by CD36 ligation on monocytes. J Exp Med. 2011;208(5):1083-1092.

116. Hosono K, et al. Roles of prostaglandin E2-EP3/ EP4 receptor signaling in the enhancement of lymphangiogenesis during fibroblast growth factor-2-induced granulation formation. Arterioscler Thromb Vasc Biol. 2011;31(5):1049-1058.

117. Huggenberger R, et al. Stimulation of lymphangiogenesis via VEGFR-3 inhibits chronic skin inflammation. J Exp Med. 2010;207(10):2255-2269.

118. Halin C, et al. Inhibition of chronic and acute skin inflammation by treatment with a vascular endothelial growth factor receptor tyrosine kinase inhibitor. Am J Pathol. 2008;173(1):265-277.

119. Baluk P, et al. Pathogenesis of persistent lymphatic vessel hyperplasia in chronic airway inflammation. J Clin Invest. 2005;115(2):247-257.

120. Huggenberger R, et al. An important role of lymphatic vessel activation in limiting acute inflammation. Blood. 2011;117(17):4667-4678.

121. Kim H, Kataru RP, Koh GY. Regulation and implications of inflammatory lymphangiogenesis. Trends in Immunology. 2012;33(7):350-356.

122. Karnezis T, et al. VEGF-D promotes tumor metastasis by regulating prostaglandins produced by the collecting lymphatic endothelium. Cancer Cell.2012; 21(2):181-195.

123. Luo Y, et al. Rapamycin inhibits lymphatic endothelial cell tube formation by downregulating vascular endothelial growth factor receptor 3 protein expression. Neoplasia. 2012;14(3):228-237.

124.Schulz MMP, et al. Phenotype-based high-content chemical library screening identifies statins as inhibitors of in vivo lymphangiogenesis. Proc Natl Acad Sci U S A. 2012;109(40):E2665-E2674.

125.Tammela T, et al. Therapeutic differentiation and maturation of lymphatic vessels after lymph node dissection and transplantation. Nat Med. 2007; 13(12):1458-1466.

126. Yoon Y-S, et al. VEGF-C gene therapy augments postnatal lymphangiogenesis and ameliorates secondary lymphedema. J Clin Invest. 2003;111(5):717-725.

127. Veikkola T, et al. Signalling via vascular endothelial growth factor receptor-3 is sufficient for lymphangiogenesis in transgenic mice. EMBO J. 2001; 20(6):1223-1231.

128. Rissanen TT, et al. VEGF-D is the strongest angiogenic and lymphangiogenic effector among VEGFs delivered into skeletal muscle via adenoviruses. Circulation. 2003;92(10):1098-1106.

129. Saaristo A, et al. Lymphangiogenic gene therapy with minimal blood vascular side effects. J Exp Med. 2002;196(6):719-730.

130. Hartiala P, Saaristo AM. Growth factor therapy and autologous lymph node transfer in lymphedema. Trends Cardiovasc Med. 2010;20(8):249-253.

131.Lähteenvuo $M$, et al. Growth factor therapy and autologous lymph node transfer in lymphedema. Circulation. 2011;123(6):613-620.

132.Greene AK, Grant FD, Slavin SA. Lower-extremity lymphedema and elevated body-mass index. N Engl J Med. 2012;366(22):2136-2137.

133. Lim HY, et al. Lymphatic vessels are essential for the removal of cholesterol from peripheral tissues by SR-BI-mediated transport of HDL. Cell Metabolism. 2013;17(5):671-684.

134. Martel C, et al. Lymphatic vasculature mediates macrophage reverse cholesterol transport in mice. J Clin Invest. 2013;123(4):1571-1579.

135. Sabine A, et al. Mechanotransduction, PROX1, and FOXC2 cooperate to control connexin 37 and calcineurin during lymphatic-valve formation. Dev Cell. 2012;22(2):430-445.

136. Krebs R, et al. Critical role of VEGF-C/VEGFR-3 signaling in innate and adaptive immune responses in experimental obliterative bronchiolitis. Am J Pathol. 2012;181(5):1607-1620.

137. Alitalo AK, et al. VEGF-C and VEGF-D blockade inhibits inflammatory skin carcinogenesis. Cancer
Res. 2013;73(14):4212-4221.

138. He Y, et al. Vascular endothelial cell growth factor receptor 3-mediated activation of lymphatic endothelium is crucial for tumor cell entry and spread via lymphatic vessels. Cancer Res. 2005; 65(11):4739-4746.

139. He Y, et al. Suppression of tumor lymphangiogenesis and lymph node metastasis by blocking vascular endothelial growth factor receptor 3 signaling. J Natl Cancer Inst. 2002;94(11):819-825.

140.Yang H, et al. Soluble vascular endothelial growth factor receptor-3 suppresses lymphangiogenesis and lymphatic metastasis in bladder cancer. Mol Cancer. 2011;10:36.

141.Lin J, et al. Inhibition of lymphogenous metastasis using adeno-associated virus-mediated gene transfer of a soluble VEGFR-3 decoy receptor. Cancer Res. 2005;65(15):6901-6909.

142.Kashima K, et al. Inhibition of lymphatic metastasis in neuroblastoma by a novel neutralizing antibody to vascular endothelial growth factor-D. Cancer Science. 2012;103(12):2144-2152.

143. Roberts N, et al. Inhibition of VEGFR-3 activation with the antagonistic antibody more potently suppresses lymph node and distant metastases than inactivation of VEGFR-2. Cancer Res. 2006; 66(5):2650-2657.

144.Pytowski B, et al. Complete and specific inhibition of adult lymphatic regeneration by a novel VEGFR-3 neutralizing antibody. J Natl Cancer Inst. 2005; 97(1):14-21.

145. Shimizu K, et al. Suppression of VEGFR-3 signaling inhibits lymph node metastasis in gastric cancer. Cancer Sci. 2004;95(4):328-333.

146.Alam A, et al. SAR131675, a potent and selective VEGFR-3-TK inhibitor with antilymphangiogenic, antitumoral, and antimetastatic activities. Mol Cancer Ther. 2012;11(8):1637-1649.

147. Heckman CA, et al. The tyrosine kinase inhibitor cediranib blocks ligand-induced vascular endothelial growth factor receptor-3 activity and lymphangiogenesis. Cancer Res. 2008;68(12):4754-4762.

148.Zhuo W, et al. The CXCL12-CXCR4 chemokine pathway: a novel axis regulates lymphangiogenesis. Clin Cancer Res. 2012;18(19):5387-5398.

149. Tammela T, et al. Photodynamic ablation of lymphatic vessels and intralymphatic cancer cells prevents metastasis. Sci Transl Med. 2011;3(69):69ra11.

150 .Honkonen KM, et al. Lymph node transfer and perinodal lymphatic growth factor treatment for lymphedema. Ann Surg. 2013;257(5):961-967. 